\title{
Salvaged single-unit cord blood transplantation for 26 patients with hematologic malignancies not in remission
}

\author{
W. Yao ${ }^{1,2}$, C.C. Zheng ${ }^{2}$, H.L. Liu ${ }^{2}$, L.Q. Geng ${ }^{2}$, B.L. Tang ${ }^{2}$, J. Tong ${ }^{2}$, X.Y. Zhu ${ }^{2}$, K.D. Song ${ }^{2}$ \\ P. Qiang ${ }^{2}$ and Z.M. Sun ${ }^{1,2}$ \\ ${ }^{1}$ Shandong University, School of Medicine, Jinan, China \\ ${ }^{2}$ Department of Hematology, Anhui Provincial Hospital, Anhui Medical University, Hefei, China
}

\begin{abstract}
Treatments for patients with hematologic malignancies not in remission are limited, but a few clinical studies have investigated the effects of salvaged unrelated cord blood transplantation (CBT). We retrospectively studied 19 patients with acute leukemia, 5 with myelodysplastic syndrome (MDS with refractory anemia with excess blasts [RAEB]), and 2 with non-Hodgkin's lymphoma who received 1 CBT unit $\leq 2$ loci human leukocyte antigen (HLA)-mismatched after undergoing myeloablative conditioning regimens between July 2005 and July 2014. All of them were in non-remission before transplantation. The infused total nucleated cell (TNC) dose was 4.07 (range $2.76-6.02$ ) $\times 10^{7} / \mathrm{kg}$ and that of $\mathrm{CD} 34^{+}$stem cells was 2.08 (range $0.99-$ $8.65) \times 10^{5} / \mathrm{kg}$. All patients were engrafted with neutrophils that exceeded $0.5 \times 10^{9} / \mathrm{L}$ on median day +17 (range $14-37$ days) and had platelet counts of $>20 \times 10^{9} / \mathrm{L}$ on median day +35 (range 17-70 days). Sixteen patients $(61.5 \%)$ experienced preengraftment syndrome (PES), and six (23.1\%) patients progressed to acute graft-versus-host disease (GVHD). The cumulative incidence rates of II-IV acute GVHD and chronic GVHD were $50 \%$ and $26.9 \%$, respectively. After a median follow-up of 27 months (range 5-74), 14 patients survived and 3 relapsed. The estimated 2-year overall survival (OS), disease-free survival (DFS), and non-relapse mortality (NRM) rates were $50.5 \%, 40.3 \%$, and $35.2 \%$, respectively. Salvaged CBT might be a promising modality for treating hematologic malignancies, even in patients with a high leukemia burden.
\end{abstract}

Key words: Umbilical cord blood transplantation; Hematologic malignancies; Non-remission

\section{Introduction}

Patients with hematologic malignancies not in remission have very poor outcomes, and allogeneic hematopoietic stem cell transplantation (allo-HSCT) is the only possibly effective treatment. However, only $25 \%$ of patients have a human-leukocyte antigen (HLA)-identical sibling. In China, the chance of having an HLA-identical sibling is very low owing to the one-child policy. Haploidentical transplantation is dependent on donor availability and post-transplantation adoptive cellular immunotherapy, but it may be complicated by a high risk of graft failure and relapse (1). Umbilical cord blood transplantation (CBT) has the advantages of being rapidly available, and having a lower incidence of graftversus-host disease (GVHD) and less strict HLA-matching requirements owing to lower numbers and more immature $\mathrm{T}$ lymphocytes. In addition, CBT procedures can safely provide a strong graft versus leukemia/lymphoma (GvL) effect $(2,3)$, especially for high-risk hematologic malignancies, and a high disease-free survival rate (4). For adults, registry-based studies have established CBT as a safe and feasible alternative to bone marrow transplantation (BMT) when a matched sibling donor is not available $(5,6)$. However, information on disease-specific outcomes is limited, in particular for patients who are not in remission before CBT. This report describes 26 patients with myeloid and lymphoid malignancies who were not in remission at the time of transplantation and who were treated with myeloablative regimens in preparation for CBT at our center. They were followed for a median of 27 months from the date of transplantation.

\section{Patients and Methods}

\section{Patients}

Twenty-six consecutive patients underwent allogeneic transplantation in our center using one unrelated cord blood unit between July 2005 and July 2014. Their

Correspondence: Zimin Sun: <zmsun_vip@163.com>. 
demographic and clinical details are shown in Tables 1 and 2. All patients had been diagnosed with hematologic malignancies. They were not in remission, defined as more than $5 \%$ blasts in bone marrow (BM). They all lacked a $5 / 6$ or fully HLA-matched related or unrelated donor. Their median age was 13 years (range 6-32 years), and their median weight before transplantation was $45 \mathrm{~kg}$ (range 18-73 kg).

\section{Transplantation procedures}

All patients underwent a myeloablative pre-transplantation conditioning regimen and $1 \mathrm{CBT}$ unit from public $\mathrm{CB}$ banks in China. The myeloablative conditioning regimens were classified into 3 groups: 1) total body irradiation (TBI)-based regimens (TBI of 3 Gy twice daily on days -7 and -6 , cytosine arabinoside [Ara-C] $2 \mathrm{~g} / \mathrm{m}^{2}$ twice daily on days -5 and -4 , and cyclophosphamide [Cy] $60 \mathrm{mg} / \mathrm{kg}$ once daily on days -3 and $-2 \pm$ carmustine [BCNU] $250 \mathrm{mg} / \mathrm{m}^{2}$ on day -5); 2) Ara-c $2 \mathrm{~g} / \mathrm{m}^{2}$ twice daily on days -9 and -8 and busulfan $(\mathrm{Bu})+\mathrm{Cy} \pm \mathrm{BCNU}$ at the same dosages as the previous regimen; or 3) Bu $4 \mathrm{mg} / \mathrm{kg}$ once daily on days -7 to -4 , and Cy $60 \mathrm{mg} / \mathrm{kg}$ once daily on days -3 and -2 . In patients with myeloid malignancies, granulocyte colony-stimulating factor (G-CSF) was infused before cytarabine. The GVHD prophylaxis consisted of intravenous ciclosporin (CsA; Novartis,
Switzerland) and mycophenolate mofetil (MMF; Roche, Switzerland). CsA was administered intravenously at a dose of $3 \mathrm{mg} / \mathrm{kg}$ daily from day -1 , and MMF was administered orally from day +1 at a dose of 25 or $30 \mathrm{mg} / \mathrm{kg}$ daily. G-CSF was used from day +6 to facilitate neutrophil engraftment until the white blood cell (WBC) count increased to at least $4 \times 10^{9} / \mathrm{L}$ for 2 consecutive days. Infection prophylaxis consisted of oral itraconazole (Janssen, China) and aciclovir (GlaxoSmithKline, UK), followed by sulfamethoxazole (Baiyunshan, China) to prevent Pneumocystis jirovecii infection.

\section{Assessment of chimerism, engraftment, and GVHD}

Confirmation of donor engraftment was made either by cytogenetics for sex-mismatched transplants or by chimerism analysis of flow-sorted peripheral blood $\mathrm{T}$ cells and nucleated marrow cells. Polymerase chain reaction (PCR) of highly polymorphic short tandem repeat (STR) units was performed. Complete donor chimerism was defined by the lack of a previously determined recipientspecific STR on polyacrylamide gel analysis. These assays could detect mixed chimerism if more than $5 \%$ donor or recipient cells were present. The day of myeloid engraftment was defined as the first of 3 consecutive days when the absolute neutrophil count exceeded $0.5 \times 10^{9} / \mathrm{L}$. The day of platelet engraftment was defined as the day

Table 1. Patient and transplant characteristics.

\begin{tabular}{lc}
\hline Characteristic & Value \\
\hline Age (years), median (range) & $13(6-32)$ \\
Sex (male/female), $\mathrm{n}$ & $15 / 11$ \\
Body weight $(\mathrm{kg})$, median (range) & $45(18-73)$ \\
Diagnosis $(\mathrm{n})$ & \\
AML & 7 \\
ALL & 11 \\
AMLL & 1 \\
MDS (RAEB) & 5 \\
NHL & 2 \\
HLA matching $(\mathrm{n})$ & \\
$6 / 6$ & 7 \\
$5 / 6$ & 12 \\
$4 / 6$ & 7 \\
TNC $\left(\times 10^{7} / \mathrm{kg}\right)$, median (range) & $7.07(2.76-6.02)$ \\
CD34 $\left(\times 10^{5} / \mathrm{kg}\right)$, median (range) & \\
Conditioning $(\mathrm{n})$ & $2.08(0.99-8.65)$ \\
TBI + Ara-c + Cy \pm BCNU & 14 \\
Ara-c + Bu + Cy \pm BCNU & 11 \\
Bu+ Cy & 1 \\
GVHD prophylaxis & $\mathrm{CsA}+\mathrm{MMF}$ \\
\hline
\end{tabular}

AML: acute myelogenous leukemia; ALL: acute lymphocytic leukemia; AMLL: acute mixed lineage leukemia; MDS: myelodysplastic syndrome; RAEB: refractory anemia with excess blast; NHL: nonHodgkin's lymphoma; HLA: human leukocyte antigen; TNC: total number of nucleated cells; TBI: total body irradiation; Ara-c: cytarabine; Cy: cyclophosphamide; BCNU: carmustine; Bu: busulfan; CsA: ciclosporin A; MMF: mycophenolate mofetil; GVHD: graft-versus-host disease. 
Table 2. Characteristics of each patient in detail.

\begin{tabular}{|c|c|c|c|c|}
\hline Patient & Disease type & Cytogenetics & $\begin{array}{l}\text { BM blasts at } \\
\text { CBT }\end{array}$ & $\begin{array}{c}\text { Disease status at } \\
\text { CBT }\end{array}$ \\
\hline$\# 1$ & MDS (RAEB-II) & Poor & $18 \%$ & Untreated \\
\hline$\# 2$ & AML & Intermediate & $92 \%$ & Refractory relapse \\
\hline$\# 3$ & ALL & Intermediate & $88 \%$ & Refractory relapse \\
\hline$\# 4$ & AML & Intermediate & $87 \%$ & Refractory relapse \\
\hline$\# 5$ & AMLL & Poor & $90 \%$ & Primary refractory \\
\hline$\# 6$ & ALL & Intermediate & $15 \%$ & Refractory relapse \\
\hline$\# 7$ & AML & Poor & $90 \%$ & Refractory relapse \\
\hline$\# 8$ & ALL & Intermediate & $70 \%$ & Refractory relapse \\
\hline$\# 9$ & ALL & Poor & $83 \%$ & Refractory relapse \\
\hline$\# 10$ & T-lymphoblastic lymphoma & Intermediate & $35 \%$ & Refractory relapse \\
\hline$\# 11$ & ALL & Intermediate & $28 \%$ & Refractory relapse \\
\hline \#12 & MDS (RAEB-II) & Poor & $17 \%$ & Untreated \\
\hline$\# 13$ & MDS (RAEB-II) & Poor & $13 \%$ & Untreated \\
\hline$\# 14$ & ALL & Intermediate & $73 \%$ & Refractory relapse \\
\hline \#15 & AML & Unknown & $14.5 \%$ & Refractory relapse \\
\hline$\# 16$ & MDS (RAEB-I) & Poor & $8.5 \%$ & Untreated \\
\hline$\# 17$ & AML & Intermediate & $84 \%$ & Refractory relapse \\
\hline \#18 & ALCL & Intermediate & $5.5 \%$ & Refractory relapse \\
\hline$\# 19$ & ALL & Intermediate & $70 \%$ & Refractory relapse \\
\hline$\# 20$ & ALL & Intermediate & $35 \%$ & Refractory relapse \\
\hline \#21 & MDS (RAEB-I) & Poor & $6.5 \%$ & Untreated \\
\hline$\# 22$ & AML & Intermediate & $85 \%$ & Refractory relapse \\
\hline \#23 & ALL & Intermediate & $79 \%$ & Refractory relapse \\
\hline$\# 24$ & ALL & Poor & $93 \%$ & Refractory relapse \\
\hline$\# 25$ & AML & Poor & $45 \%$ & Primary refractory \\
\hline \#26 & ALL & Unknown & $67 \%$ & Refractory relapse \\
\hline
\end{tabular}

BM: bone marrow; CBT: cord blood transplantation; MDS: myelodysplastic syndrome; RAEB: refractory anemia with excess blast; AML: acute myelogenous leukemia; ALL: acute lymphocytic leukemia; AMLL: acute mixed lineage leukemia; ALCL: anaplastic large cell lymphoma.

when the absolute platelet count exceeded $20 \times 10^{9} / \mathrm{L}$ without platelet transfusion. Both acute and chronic GVHD (aGVHD and cGVHD) were diagnosed and graded based on published criteria $(7,8)$.

\section{Statistical analysis}

The endpoints of this study were engraftment, aGVHD and cGVHD, infectious complications, non-relapse mortality (NRM), relapse rate, disease-free survival (DFS), and overall survival (OS). Survival rates were calculated by the Kaplan-Meier method with the log-rank test. The cumulative incidents rates of NRM, relapse, aGVHD, and cGVHD were computed to determine the presence of competing risks (9). $\mathrm{P}<0.05$ was considered to indicate statistical significance in all analyses.

\section{Results}

Engraftment, transplantation toxicity, and infections All patients were engrafted with neutrophils that exceeded $0.5 \times 10^{9} / \mathrm{L}$ on median day +17 (range 14-37 days) and had platelet counts $>20 \times 10^{9} / \mathrm{L}$ on median day +35 (range 17-70 days). Three patients died before platelet engraftment after CBT because of severe GVHD and intestinal infection. By day 21 post CBT these patients had all converted to full donor chimerism (FDC). Sixteen (61.5\%) patients experienced pre-engraftment syndrome (PES), and $6(23.1 \%)$ patients progressed to aGVHD.

Severe pneumonia and intestinal infection occurred in six patients and eventually led to death. Sepsis was documented in five patients but was controlled by antibiotics during the agranulocytosis period after CBT. Seven patients developed cytomegalovirus (CMV) viremia (CMV-DNA $>10^{3}$ copies $/ \mathrm{mL}$ ) but were negative after antiviral treatment. None developed CMV-associated diseases. Three patients experienced hemorrhagic cystitis and were cured by adequate hydration and urine alkalinization.

\section{GVHD}

The cumulative incidence of aGVHD was 50\% (95\% confidence interval $[\mathrm{CI}]=31.0-69.0 \%$, and grade II-IV 

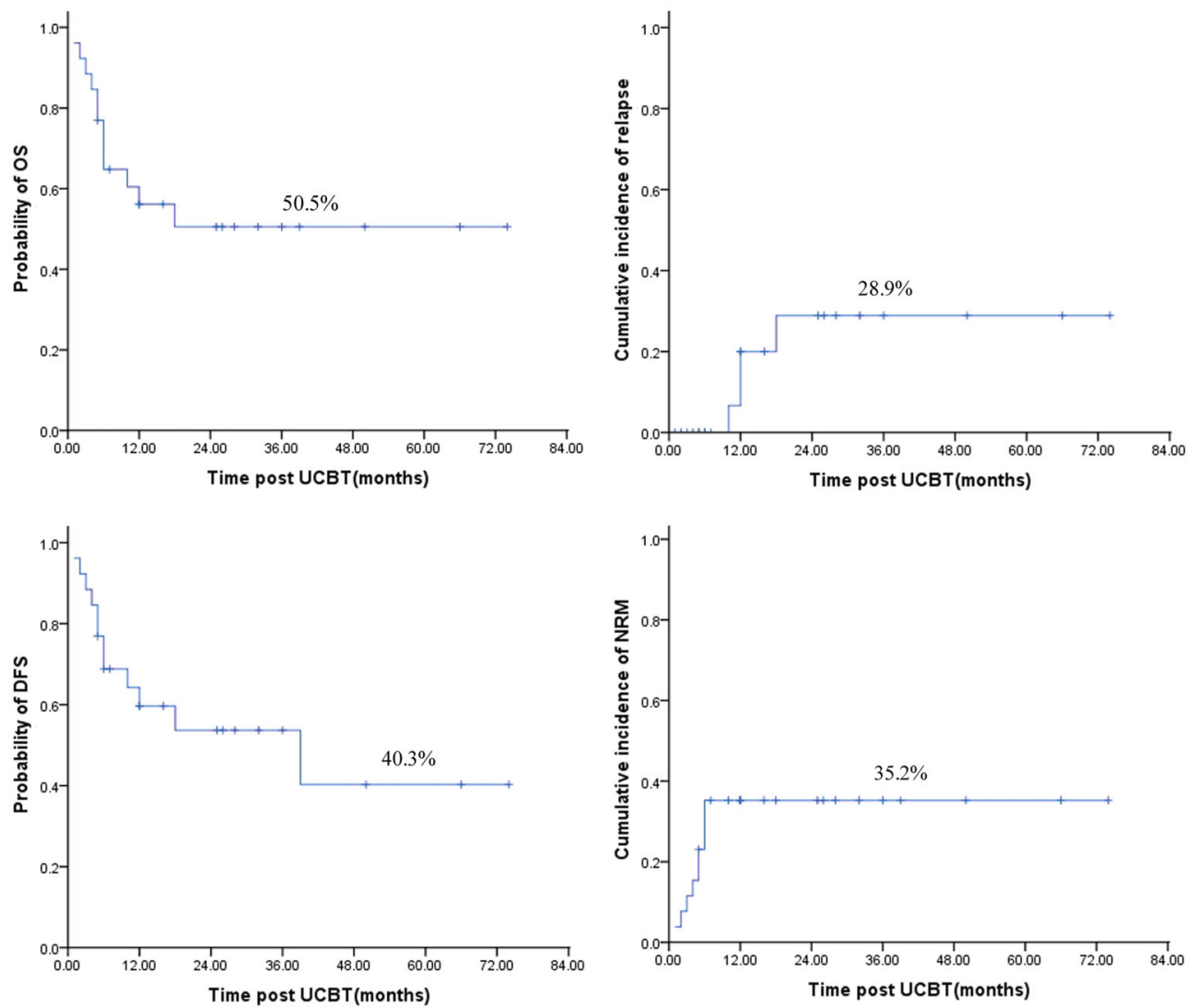

Figure 1. Overall survival (OS), disease-free survival (DFS), relapse and non-relapse mortality (NRM) in patients undergoing cord blood transplantation. UCBT: umbilical cord blood transplantation.

aGVHD was diagnosed in $26.9 \%$ (95\% Cl=9.9-43.9\%). Thirteen patients developed aGVHD. Three patients died of grade IV aGVHD before day +100 . In the remaining 10 patients, aGVHD was under control after treatment with CsA or tacrolimus combined with methylprednisolone and MMF. With a median follow-up of 27 months (range 5-74 months) for surviving patients, the cumulative incidence of cGVHD was $26.9 \%(95 \% \mathrm{Cl}=9.9-43.9 \%)$, and there was no extensive cGVHD.

\section{Outcome}

As of July 2014, the median follow-up was 27 months (range 5-74 months). In all, 14 patients survived who were in sustained complete remission (CR) and 12 died, 3 from relapse and 9 from NRM (6 from severe infection, 3 from aGVHD). The estimated 2-year OS, DFS, relapse, and NRM rates were $50.5 \%(95 \% \mathrm{Cl}=31.3-69.7 \%), 40.3 \%$ (95\% Cl=21.4-59.2\%), 28.9\% (95\% Cl=11.5-46.3\%), and $35.2 \%(95 \% \mathrm{Cl}=16.8-53.6 \%)$, respectively (Figure 1).

\section{Discussion}

Despite the important growth in CBT procedures worldwide, especially for adult patients with high-risk hematologic malignancies $(4,10,11)$, our study is unique in that none of the patients enrolled were in remission at the time of transplantation. Although we assessed a highrisk cohort, the estimated 2-year OS and DFS were 50.5\% and $40.3 \%$, respectively, which are comparable with those of other studies (though only a subset of the patients enrolled were in advanced stages before CBT) $(4,10,11)$.

Unrelated donor CBT is associated with a significant engraftment failure rate $(10,12,13)$. This unfavorable outcome is due to a low cell dose infused and the frequent HLA disparity between the donor and recipient. In our study, as in those carried out at the University of Tokyo (14) and in Spain (11), hematopoietic recovery in CBT recipients was rapid. No primary or secondary graft failure was observed, and engraftments were achieved within 21 days in all engrafted patients. The intensification of conditioning by administering additional cytarabine in the setting of TBI-CY or BU-CY, which remain the most common myeloablative conditioning regimens for allogeneic HSCT without the use of an anti-T cell antibody, was sufficiently effective to eradicate malignant cells and achieve engraftment. In addition, we combined cytarabine with G-CSF infusion in patients with myeloid malignancies based on the hypothesis that G-CSF increases the susceptibility of myeloid leukemic cells to cytarabine $(15,16)$. 
Three risk factors for grade II-IV aGVHD after CBT were identified in multiple regression analysis: use of $2 \mathrm{CB}$ units, use of non-myeloablative conditioning, and absence of antithymocyte globulin in the conditioning regimen (17). As was previously reported $(4,11,17)$, we confirmed a relatively low incidence of aGVHD/cGVHD after CBT despite the absence of antithymocyte globulin. This may be related to the use of one CB unit or to intensified myeloablative conditioning. In addition, MMF may be more effective than MTX in GVHD prophylaxis.

Regarding NRM, we observed a higher rate $(35.2 \%)$ than that reported by the University of Tokyo study (15\%) (3). However, other studies reported cumulative NRM incidence rates of $30 \%$ to $60 \%(6,11,18-22)$. The most important reason was disease status at transplantation, which was associated with extensive prior treatment and poor tolerance to transplant-associated toxicities that likely increased transplantation related mortality (TRM). We also noted higher mortality due to infection. Future investigations should focus on more effective preventive

\section{References}

1. Ballen KK, Koreth J, Chen YB, Dey BR, Spitzer TR. Selection of optimal alternative graft source: mismatched unrelated donor, umbilical cord blood, or haploidentical transplant. Blood 2012; 119: 1972-1980, doi: 10.1182/ blood-2011-11-354563.

2. Barker JN, Weisdorf DJ, DeFor TE, Blazar BR, McGlave PB, Miller JS, et al. Transplantation of 2 partially HLAmatched umbilical cord blood units to enhance engraftment in adults with hematologic malignancy. Blood 2005; 105: 1343-1347, doi: 10.1182/blood-2004-07-2717.

3. Ballen KK, Spitzer TR, Yeap BY, McAfee S, Dey BR, Attar $\mathrm{E}$, et al. Double unrelated reduced-intensity umbilical cord blood transplantation in adults. Biol Blood Marrow Transplant 2007; 13: 82-89, doi: 10.1016/j.bbmt.2006. 08.041.

4. Mori T, Tanaka M, Kobayashi T, Ohashi K, Fujisawa S, Yokota A, et al. Prospective multicenter study of single-unit cord blood transplantation with myeloablative conditioning for adult patients with high-risk hematologic malignancies. Biol Blood Marrow Transplant 2013; 19: 486-491, doi: 10.1016/j.bbmt.2012.12.007.

5. Rocha V, Labopin M, Sanz G, Arcese W, Schwerdtfeger R, Bosi A, et al. Transplants of umbilical-cord blood or bone marrow from unrelated donors in adults with acute leukemia. N Engl J Med 2004; 351: 2276-2285, doi: 10.1056/NEJMoa041469.

6. Laughlin MJ, Eapen M, Rubinstein P, Wagner JE, Zhang MJ, Champlin RE, et al. Outcomes after transplantation of cord blood or bone marrow from unrelated donors in adults with leukemia. N Engl J Med 2004; 351: 2265-2275, doi: 10.1056/NEJMoa041276.

7. Przepiorka D, Weisdorf D, Martin P, Klingemann HG, Beatty P, Hows J, et al. 1994 Consensus Conference on Acute GVHD Grading. Bone Marrow Transplant 1995; 15: 825-828. and therapeutic measures to manage infectious complications and improve outcomes.

The relapse rate was low and similar to those previously reported for patients with high-risk hematologic malignancies in the setting of CBT (10); it was even superior to that of haploidentical (40\%) (23) and related or unrelated peripheral stem cell transplantation (32\%) (24), confirming the high antileukemic efficacy of the procedure. Hence, OS and DFS were mainly influenced by NRM and the associated risk factors.

In conclusion, single-unit CBT with myeloablative conditioning can be carried out with acceptable toxicity and TRM and increases long-term remission in patients with hematologic malignancies who were not previously in remission. The limitations of this study are that it was a retrospective analysis of a small number of patients with relatively short follow-up periods in the survivors. Additional large-scale studies with long-term follow-up are needed to better evaluate the efficacy of this procedure.

8. Shulman HM, Sullivan KM, Weiden PL, McDonald GB, Striker GE, Sale GE, et al. Chronic graft-versus-host syndrome in man. A long-term clinicopathologic study of 20 Seattle patients. Am J Med 1980; 69: 204-217, doi: 10.1016/0002-9343(80)90380-0.

9. Klein JP, Rizzo JD, Zhang MJ, Keiding N. Statistical methods for the analysis and presentation of the results of bone marrow transplants. Part I: unadjusted analysis. Bone Marrow Transplant 2001; 28: 909-915, doi: 10.1038/ sj.bmt. 1703260.

10. Lekakis L, Giralt S, Couriel D, Shpall EJ, Hosing C, Khouri IF, et al. Phase II study of unrelated cord blood transplantation for adults with high-risk hematologic malignancies. Bone Marrow Transplant 2006; 38: 421-426, doi: 10.1038/ sj.bmt. 1705467.

11. Sanz J, Sanz MA, Saavedra S, Lorenzo I, Montesinos P, Senent $\mathrm{L}$, et al. Cord blood transplantation from unrelated donors in adults with high-risk acute myeloid leukemia. Biol Blood Marrow Transplant 2010; 16: 86-94, doi: 10.1016/ j.bbmt.2009.09.001.

12. Stevens CE, Scaradavou A, Rubinstein P. Cord blood transplantation to adult patients: A single-bank's experience. Biol Blood Marrow Transplant 2004; 10: 733-739, doi: 10.1016/j.bbmt.2004.06.011.

13. Kurtzberg J, Carter SL, Baxter-Lowe LA, Wagner JE, Baxter-Lowe LA, Wall D, et al. Results of the cord blood transplantation study (COBLT): Clinical outcomes of 193 unrelated donor umbilical cord blood transplantation in pediatric patients with malignant conditions. Biol Blood Marrow Transplant 2015; 11 (Suppl 1): Abstract No. 6.

14. Ooi J, Takahashi S, Tomonari A, Tsukada N, Konuma T, Kato $\mathrm{S}$, et al. Unrelated cord blood transplantation after myeloablative conditioning in adults with acute myelogenous leukemia. Biol Blood Marrow Transplant 2008; 14: 1341-1347, doi: 10.1016/j.bbmt.2008.09.007. 
15. Lowenberg B, van Putten W, Theobald M, Gmur J, Verdonck L, Sonneveld $P$, et al. Effect of priming with granulocyte colony-stimulating factor on the outcome of chemotherapy for acute myeloid leukemia. $N$ Engl J Med 2003; 349: 743-752, doi: 10.1056/NEJMoa025406.

16. Amadori S, Suciu S, Jehn U, Stasi R, Thomas X, Marie JP, et al. Use of glycosylated recombinant human G-CSF (lenograstim) during and/or after induction chemotherapy in patients 61 years of age and older with acute myeloid leukemia: final results of AML-13, a randomized phase-3 study. Blood 2005; 106: 27-34, doi: 10.1182/blood-2004-09-3728.

17. Atsuta $\mathrm{Y}$, Suzuki R, Nagamura-Inoue T, Taniguchi S, Takahashi S, Kai S, et al. Disease-specific analyses of unrelated cord blood transplantation compared with unrelated bone marrow transplantation in adult patients with acute leukemia. Blood 2009; 113: 1631-1638, doi: 10.1182/ blood-2008-03-147041.

18. Kumar P, DeFor TE, Brunstein C, Barker JN, Wagner JE, Weisdorf DJ, et al. Allogeneic hematopoietic stem cell transplantation in adult acute lymphocytic leukemia: impact of donor source on survival. Biol Blood Marrow Transplant 2008; 14: 1394-1400, doi: 10.1016/j.bbmt.2008.09.021.

19. Cornetta K, Laughlin M, Carter S, Wall D, Weinthal J, Delaney $\mathrm{C}$, et al. Umbilical cord blood transplantation in adults: results of the prospective Cord Blood Transplantation (COBLT). Biol Blood Marrow Transplant 2005; 11: 149-160, doi: 10.1016/j.bbmt.2004.11.020.
20. Matsumura T, Kami M, Yamaguchi T, Yuji K, Kusumi E, Taniguchi $\mathrm{S}$, et al. Allogeneic cord blood transplantation for adult acute lymphoblastic leukemia: retrospective survey involving 256 patients in Japan. Leukemia 2012 26: 1482-1486, doi: 10.1038/leu.2012.11.

21. Long GD, Laughlin M, Madan B, Kurtzberg J, Gasparetto C Morris A, et al. Unrelated umbilical cord blood transplantation in adult patients. Biol Blood Marrow Transplant 2003; 9: 772-780, doi: 10.1016/j.bbmt.2003.08.007.

22. Oran B, Wagner JE, DeFor TE, Weisdorf DJ, Brunstein CG. Effect of conditioning regimen intensity on acute myeloid leukemia outcomes after umbilical cord blood transplantation. Biol Blood Marrow Transplant 2011; 17: 1327-1334, doi: 10.1016/j.bbmt.2011.01.007.

23. Solomon SR, Sizemore CA, Sanacore M, Zhang X, Brown $S$, Holland HK, et al. Haploidentical transplantation using $T$ cell replete peripheral blood stem cells and myeloablative conditioning in patients with high-risk hematologic malignancies who lack conventional donors is well tolerated and produces excellent relapse-free survival: results of a prospective phase II trial. Biol Blood Marrow Transplant 2012; 18: 1859-1866, doi: 10.1016/j.bbmt.2012.06.019.

24. Denz U, Bertz H, Ihorst G, Wasch R, Finke J. Improved outcome in relapsed and refractory myeloid malignancies for unrelated vs related donor allogeneic peripheral blood-derived hematopoietic cell transplantation. Bone Marrow Transplant 2010; 45: 1309-1315, doi: 10.1038/bmt.2009.341. 